\title{
ESTIMACIÓN DE LA MORTALIDAD INTERCENSAL MEDIANTE EL USO DE FACTORES DE SEPARACIÓN*
}

\author{
JUAN CARlos LeRda** \\ Center of Population Studies, Harvard University
}

\section{INTRODUCCIÓN}

ESTE TRABAjo constituye un análisis teórico del problema tradicional de construir una "tabla de vida" intercensal o sea el problema de cómo desarrollar a partir del conjunto ${ }_{n} L_{x}$ el conjunto correspondiente $l_{x}$.

Es bien sabido que si se dispone de dos censos comparables, correspondientes a una población cerrada clasificada por sexo y edad, existe una variedad de técnicas demográficas disponibles para construir una "tabla de vida" representativa del nivel medio de la mortalidad durante el período intercensal [1], [2], [3]. De manera general, las principales etapas involucradas en las metodologías usadas corrientemente son:

1) Calcular las relaciones de supervivencia observadas ${ }_{n} P_{x}{ }^{\prime}$, por cohorte correspondientes al período intercensal;

2) Ajustar estos valores corregidos por irregularidades según se supone debido a la inexactitud de los datos básicos;

3) Transformar estas tasas ajustadas de supervivencia en el conjunto de valores ${ }_{n} L_{x}$ correspondientes;

4) Transformar el conjunto ${ }_{n} L_{x}$ en el correspondiente $l_{x}$ y finalmente;

5) Completar las funciones biométricas restantes de la "tabla de vida" siguiendo las definiciones convencionales.

- Trabajo presentado a la Comisión sobre Demografía Matemática de la conferencia de Población de Lieja a solicitud del profesor N. Keyfitz organizador de dicha comisión, bajo el título: "How to Make a Life Table When Two Age Distributions Are Given (or, How to Transform $l_{x}$ into $l_{r}$ )." Una primera versión de las ideas aquí contenidas fue presentada en el curso "Comparative Demography of Latin America" dictado por el profesor Eduardo Arriaga, Department of Demography, Berkeley, 1971-1972. El autor agradece a ambos profesores por sus valiosas observaciones y al Dr. E. Bergman, Director Asistente del Center for Population Studies, Harvard University, por las facilidades administrativas que hicieron posible la versión inglesa de este artículo. Un agradecimiento especial para Raúl de la Peña que tradujo el artículo al español.

* Estudiante del programa de doctorado, Departamento de Economía, Universidad de Harvard, Cambridge, U.S.A. 
Como se indicó al principio, este trabajo centra su atención en la cuarta etapa, aunque los párrafos iniciales se dedicarán a la revisión de unos pocos y bien conocidos resultados en relación a la primera y tercera etapas como punto de partida de nuestro tema de interés.

\section{LA PRIMERA META: LA DISTRIBUCIÓN ${ }_{n} L_{x}$}

Si la mortalidad fuera la causa única de la disminución de la población por cohortes durante el período intercensal $o$ a $n$, entonces las relaciones de supervivencia:

$$
{ }_{n} P_{x}^{o, n}=\frac{{ }_{n} N_{x+n}^{n}}{{ }_{n} N_{x}^{o}}
$$

representan el concepto equivalente en una tabla de vida correspondiente a algún punto intermedio entre $o$ y $n$. Sin duda, cada ${ }_{n} P_{x}^{o, n}$ representa un nivel medio de mortalidad intercensal por cohortes y no necesariamente tiene que estar referida al mismo momento. Sin embargo, para simplificar se considera el supuesto usual de que todos ellos se refieren al punto $n / 2$.

Antes de pasar adelante, nótese que hemos supuesto censos comparables y por lo tanto, no debemos inquietarnos por las dificultades usuales asociadas con el ajuste de las relaciones de supervivencia observadas: las técnicas demográficas convencionales permiten solucionar el problema. [2], [3], [4]

Bajo las primeras condiciones podemos escribir:

$$
\left({ }_{n} P_{x}=\frac{N_{x+n}}{{ }_{n} N_{x}}\right)=\left({ }_{n} P_{x}^{n / 2}=\frac{{ }_{n}^{n / 2}{ }_{x+n}^{n / 2}}{{ }_{n} L_{x}^{n / 2}}\right)
$$

Con el conjunto de valores ${ }_{n} P_{x}^{n / 2}$ y eliminando el superíndice $n / 2$ una vez que se ha estimado ${ }_{n} P_{b}\left(={ }_{n} L_{o} / n \cdot l_{0}\right)$, podemos derivar el vector ${ }_{n} L_{x}$ asociado con una tabla de vida implícita referida a $t=n / 2$. Entonces, dado ${ }_{n} P_{b},{ }_{n} P_{o},{ }_{n} P_{n}, \ldots,{ }_{n} P_{x}, \ldots,{ }_{n} P_{80}(+), y$ aplicando las definiciones convencionales:

$$
\begin{aligned}
{ }_{n} L_{o}^{*} & =n \cdot l_{o} \cdot{ }_{n} P_{b} \\
{ }_{n} L_{n}^{*} & ={ }_{n} L_{o}^{*} \cdot{ }_{n} P_{o} \\
{ }_{n} L_{80}^{*} & ={ }_{n} L_{80-n}^{*} \cdot{ }_{n} P_{80-n}^{*} \\
T_{80+n}^{*} & =\frac{{ }_{n} L_{80}^{*} \cdot{ }_{n} P_{80(+)}}{\left(l-{ }_{n} P_{80(+)}\right)}
\end{aligned}
$$

tenemos la materia prima para la transformación que nos interesa. 


\section{LA RELACIÓN ENTRE ${ }_{n} L_{x}$ Y $l_{x}$}

Desde un punto de vista estrictamente matemático, estas dos funciones biométricas se relacionan a través de la bien conocida expresión:

$$
\int_{0}^{n} l(x+t) \cdot d t={ }_{n} L_{x}
$$

Así, es claro que el resultado de estas operaciones dobles (para encontrar ${ }_{n} L_{x}$ a partir de $l_{x}$, o viceversa) dependerá de la forma analítica que se supone para $l(x+t)$. Por lo tanto, cuando se formula la cuarta etapa de la metodología general, al decir "transfórmese el conjunto ${ }_{n} L_{x}$ en el conjunto correspondiente $l_{x}$ " lo que se quiere decir es que existe una correspondencia unívoca, bajo una hipótesis específica, acerca de la forma matemática de $l(x+t)$. En el contexto de este trabajo, hay una variación relacionada muy de cerca con el propósito práctico impuesto y que consiste en encontrar los valores aislados por edades para ${ }_{n} L_{x}$, generalmente dados aplicando multiplicadores de Beer [5] y estableciendo un supuesto acerca de la forma analítica de $L_{x}$, de la cual se deriva el conjunto $l_{x}$ por interpolación.

Dado que el problema de pasar de ${ }_{n} L_{x}$ a $l_{x}$ no es nuevo, han sido adelantadas varias soluciones. Se han sugerido gran variedad de fórmulas siguiendo el primer enfoque mencionado con algunos cambios en la forma matemática de $l(x+t)$ teniendo en cuenta la diferente curvatura de la función por edad [6], [2]. Ejemplos del segundo enfoque pueden encontrarse en [7], [8]. En general, el uso de tales formas matemáticas tiene ventajas obvias: aparte de sus propiedades analíticas elegantes produce resultados consistentes externamente; también, constituyen técnicas que ahorran tiempo y molestias cuando se involucran multiplicadores.

No obstante, detrás de la apariencia de consistencia externa (en realidad no es posible encontrar publicados valores crecientes de $l_{x}$ ), aquellas fórmulas pueden muy bien tener incoherencias implícitas entre las funciones clave, las que no tienen sentido desde un punto de vista demográfico.

Nos gustaría adelantar aquí la idea de estudiar la consistencia interna de las "tablas de vida" a través del concepto clave del número medio de años vividos en el intervalo $(x, x+n-1)$ por aquellos que murieron en dicho intervalo $\left.{ }_{n} a_{x}\right)$. Nuestro punto de vista es que mientras en el procedimiento convencional para construir tablas de vida corrientes (o sea a partir de tasas específicas de mortalidad por edades $\left.{ }_{x} M_{x}\right)$ observadas), el concepto ${ }_{n} a_{x}$ se incorpora explícita y cuidadosamente a través de la expresión:

$$
{ }_{n} L_{x}=n \cdot l_{x+n}+{ }_{n} a_{x} \cdot{ }_{n} d_{x}
$$

en los procedimientos indirectos, una vez que las $l_{x}$ son derivadas de las ${ }_{n} L_{x}$ utilizando las técnicas previamente mencionadas, el concepto ${ }_{n} a_{x}$ 
no aparece como un insumo explícito sino como un producto implícito. Una consecuencia importante es que las ${ }_{n} a_{x}$ implícitas, generadas por ciertas fórmulas de interpolación usadas para obtener $l_{x}$ a partir de ${ }_{n} L_{x}$ son simplemente erróneas o por lo menos contradicen la evidencia empírica. Por ejemplo, hay acuerdo general sobre el hecho empírico de que $a_{0}$ está relacionada inversamente con el nivel de la esperanza de vida al nacer, o lo que es lo mismo, directamente relacionada con el nivel de mortalidad infantil. Más aún, hay evidencia abundante de que $a_{o}$ está comprendida entre aproximadamente 0.10 para esperanzas de vida al nacer cercanas a 70 años [9] y 0.35 para esperanzas de vida al nacer reducidas [10], [11], [12]. Por ejemplo, en 1967 el factor de separación $a_{o}$ para la población total de Estados Unidos se encontró que era 0.09 , consistente con $\mathrm{e}_{o}^{o}=70.59$ años [9]. De acuerdo con todo esto, considérese la siguiente paradoja. Por procedimientos independientes fueron construidas dos "tablas de vida" para la población femenina de Chile obteniéndose los siguientes resultados:

\begin{tabular}{rlll}
\hline & $A \tilde{n} o$ & $e_{o}^{o}$ & $a_{o}$ \\
\hline II & 1907 & 29.67 & 0.07 \\
\hline
\end{tabular}

Fuente: I véase la nota 7 del texto; II véase la nota 13.

Es obvio que si juzgamos la consistencia de estos resultados como se hace usualmente, a través del indicador $\mathrm{e}_{o}^{o}$ nunca podremos encontrar lo que está sucediendo dentro de la tabla de vida. Es importante entonces establecer que mientras el resultado $a_{o}=0.68$ proviene del uso irrestricto de una ecuación simple de regresión, y siguiendo el procedimiento convencional (de ${ }_{n} M_{x}$ a ${ }_{n} q_{x}$ ) para construir una tabla de vida, el otro, o sea $a_{o}=0.07$ resulta de la insensibilidad demográfica de rígidas formas matemáticas usadas como artificios de interpolación. Sin embargo, cualquiera que sea la razón de tan extraños resultados, esperamos haber hecho clara la utilidad de ${ }_{n} a_{x}$ y particularmente de $a_{o}$, como instrumento para medir la consistencia interna de la tabla de vida. Éste es especialmente el caso cuando es construida derivando la función $l_{x}$ del conjunto ${ }_{n} L_{x}$.

Con lo anterior es suficiente en relación con ${ }_{n} a_{x}$ como índice de consistencia interna. Sin embargo, ésta es en realidad una razón atrayente para buscar un modo de derivar la función $l_{x}$ a partir de ${ }_{n} L_{x}$, de tal modo que la distribución $a_{x}$ sea tomada en cuenta explícitamente. ¿Es esto posible? Por fortuna la respuesta es afirmativa y lo que resta de este trabajo se dedica a la elaboración de un tratamiento general de este problema así como a derivar algunos casos particulares de especial importancia. 


\section{TRANSFORMACIÓN DE ${ }_{n} L_{x}$ ES $l_{x}$ A TRAVÉS DE ${ }_{n} a_{x}$}

Como ya se ha sugerido, el concepto de número medio de años vivido en un intervalo dado por aquellos que murieron en el intervalo $\left({ }_{n} a_{x}\right)$ jugará un papel clave en la solución del problema de transformar ${ }_{n} L_{x}$ en $l_{x}$. De hecho, su definición servirá para derivar los resultados que nos interesan. En relación con esto, es importante entender que ${ }_{n} a_{x}$ es un concepto definido dentro del contexto y empíricamente medido a partir de datos básicos correspondientes a la población real y observada. Por 10 tanto, lo definiremos en su forma general.

$$
{ }_{n} a_{x}=\frac{\int_{0}^{n} t \cdot N(x+t) \mu(x+t) \cdot d t}{\int_{0}^{n} N(x+t) \cdot \mu(x+t) \cdot d t}
$$

en donde:

$$
\begin{gathered}
N(x+t) \cdot d t \text { representa la población expuesta al riesgo de morir } \\
\text { entre las edades } x+t \text { y } x+t+d t, y \\
\mu(x+t) \text { expresa la fuerza de la mortalidad a la edad } x+t
\end{gathered}
$$

Es importante señalar que estamos siguiendo el enfoque sugerido por Keyfitz en un contexto algo diferente [14]; por consiguiente, se ha supuesto que una curva continua de mortalidad, llamada la fuerza de la mortalidad, se basa en las ${ }_{n} a_{x}$ observadas "y que la misma curva debe basarse en la tabla de vida".

No hay duda acerca del hecho de que la expresión IV.1 no ayuda a resolver nuestro problema en la medida en que no hagamos un supuesto sobre la forma analítica correspondiente a la distribución subyacente de población $N(x+t)$. Por lo tanto, para avanzar hacia una solución sugerimos la sustitución general:

$$
N(x+t)=F(t) l(x+t)
$$

donde $F(t)$ está sujeta solamente a la condición de que sea derivable dentro del intervalo abierto $x, x+n$. Es claro que $F(t)$ permanece como incógnita y que la solución particular dependerá en última instancia del supuesto específico que se decida hacer acerca de su forma analítica.

Por lo que se refiere a la racionalidad demógrafica de incluir $l(x+t)$ en dicha transformación, se puede argumentar que a partir del supuesto de que la misma fuerza de mortalidad rige tanto para la población observada como para la tabla de vida, cojuntamente con el de la población cerrada, tal supuesto parece teóricamente justificado. Esto es, en el presente nivel de generalidad $F(t)$ puede interpretarse como una fuerza equilibrante entre ambas funciones de densidad, la observada y la estaciona- 
ria, que toma en cuenta diferentes patrones de fecundidad. Es claro que bajo supuestos adicionales tales como mortalidad y fecundidad constantes por edad, $F(t)$ adquiere una bien conocida forma analítica, en cuyo caso, la introducción de $l(x+t)$ se justifica completamente. Finalmente, con una forma indeterminada de $F(t)$ el propósito del trabajo en esta etapa es generar una solución general de la cual se puedan derivar todos los casos particulares. Aquí solamente se mostrará la solución para unos casos de reconocido interés para la demografía.

A. Sustituyendo IV.2 en IV.1 y considerando el hecho de que $\mu(x+t)=$ $-d \ln l(x+t) / d t$, tenemos:

$$
{ }_{n} a_{x}=\frac{\int_{0}^{n} t F(t) \cdot d l(x+t)}{\int_{0}^{n} F(t) \cdot d l(x+t)}
$$

Integrando por partes y usando una extensión familiar del teorema del valor medio para integrales [15] encontramos que:

$$
\begin{array}{ll}
\text { (IV.4) }{ }_{n} a_{x}=\frac{n \cdot F(n) \cdot l(x+n)-{ }_{n} L_{x}\left[F(\theta)+F^{\prime}(z){ }_{n} A_{x}\right]}{F(n) \cdot l(x+n)-F(0) \cdot l(x)-F^{\prime}(h){ }_{n} L_{x}} \\
\text { donde: } \quad 0 \leq h \leq n ; \leq \leq \leq \leq n ; \\
\\
F^{\prime} \text { expresa la primera derivada; y } \\
{ }_{n} A_{x}=\frac{\int_{0}^{n} t \cdot l(x+t) \cdot d t}{\int_{0}^{n} l(x+t) \cdot d t}
\end{array}
$$

A partir de IV.4 y resolviendo las incógnitas:

$$
l(x+n)=\frac{F(\theta) \cdot{ }_{n} L_{x}-F(o) \cdot{ }_{n} a_{x} \cdot l(x)}{F(n) \cdot\left(n-{ }_{n} a_{x}\right)}
$$

obtenemos una aproximación a la solución general completa, la cual podría contener un término adicional en el numerador, esto es:

$$
{ }_{n} L_{x}\left[F^{\prime}(z) \cdot{ }_{n} A_{x}-F^{\prime}(h) \cdot{ }_{n} a_{x}\right] .
$$

Por supuesto, solamente sería una buena aproximación en la medida en que la diferencia entre corchetes se acerque a cero. En realidad, sería exacto cada vez que $z \approx h$ y ${ }_{n} A_{x} \approx{ }_{n} a_{x}$, lo cual puede esperarse que sea el caso excepto para los primeros y los últimos años del ciclo de vida.

$B$. Una etapa posterior podría ser imponer a la función $F(t)$ la con- 
dición adicional $F^{\prime}(t)=c F(t)$ que nos pone en contacto con la familia de las funciones exponenciales cuyo interés demográfico es conocido. Siguiendo la misma línea de razonamiento que la utilizada en la fórmula IV.5 puede demostrarse que bajo el supuesto usual se obtiene la misma aproximación. En este caso $c$ denota una constante o por lo menos que es independiente de la variable $t$. Finalmente, la solución general completa de este caso incluye en el numerador el término adicional:

$$
c F(\theta) \cdot{ }_{n} L_{x}\left({ }_{n} A_{x}-{ }_{n} a_{x}\right)
$$

$C$. Un caso especialmente importante es el de la estabilidad local, o aún más aquel donde la distribución de densidad observada corresponde a una población estable. Dado lo que hemos supuesto: $F(t)=K \exp (-r t)$, donde $K=b$, la tasa intrínseca de natalidad no aparece en nuestro resultado final, $\mathbf{y}$ en vista de que este es un caso en el cual se cumple la condición $F^{\prime}(t)=c \cdot F(t)$ podemos evitar el procedimiento convencional de integración por partes y aplicar directamente el resultado IV.5 que como se dijo es válido bajo el supuesto usual. Por lo tanto.

$$
l(x+n)=\frac{e^{-r n / 2}{ }_{n} L_{x}-{ }_{n} a_{x} l(x)}{e^{-r n} \cdot\left(n-{ }_{n} a_{x}\right)}
$$

fue obtenido al seleccionar el valor aproximado de $\theta=n / 2$. Es importante tener en cuenta que esta es una aproximación a la solución completa la cual incluye en el numerador el término adicional $r e^{-r n / 2} \cdot{ }_{n} L_{x} \cdot\left({ }_{n} a_{x}-{ }_{n} A_{x}\right)$. Como hemos visto, bajo la hipótesis usual, la exactitud de nuestra aproximación dependerá no solamente de la similitud entre ${ }_{n} A_{x}$ y ${ }_{n} a_{x}$ sino también de cuán pequeña sea la tasa intrínseca de crecimiento o de ambas cosas.

D. De este importante caso llegamos a la conclusión de que para un conjunto dado de valores de ${ }_{n} L_{x}$ el resultado final de nuestra transformación dependerá de los supuestos que se hagan con relación a: $i$ ) la tasa de crecimiento de la población, ii) la manera en que se distribuyan las muertes dentro de cada intervalo de edades. Así, si tomamos la fórmula IV.6 para $r \neq 0$ y ${ }_{n} a_{x} \neq n / 2$ hay posibilidad para tres subcasos, dos de los cuales tienen un interés especial desde el punto de vista demográfico:

$$
\text { cuando } r=o \mathrm{y}_{n} a_{x} \neq n / 2 \text { tenemos: }
$$

$$
l(x+n)=\frac{{ }_{n} L_{x}-{ }_{n} a_{x} l(x)}{n-{ }_{n} a_{x}}
$$

đque no es sólo una solución aproximada sino la exacta. Es claro que esta solución a nuestro problema podría haber sido derivada de la bien conocida expresión III.2 mediante una transformación algebraica simple (de hecho así se hizo primero cuando se nos ocurrió la idea de este tema). Sin embargo, pensamos que el método presentado aquí es preferible desde 
un punto de vista teórico: 1) porque permite visualizar con claridad como se origina de un conjunto más general de supuestos que de aquellos de los cuales es aparentemente deducido cuando se toma como punto de partida la expresión III.2; y 2) permite observar cuál es su posición dentro de un proceso lógico de deducción así como la interrelación que existe entre ésta y otras soluciones al mismo problema que se derivan de ese proceso.

$$
\begin{gathered}
\text { cuando } r=o \mathrm{y}_{n} a_{x}=n / 2 \text { tenemos: } \\
\qquad l(x+n)=\frac{2{ }_{n} L_{x}-n l(x)}{n}
\end{gathered}
$$

que para el caso de ${ }_{n} a_{x}=n / 2$ puede ser derivada obviamente de III.2, o sea la bien conocida regla de los trapecios:

$$
\begin{aligned}
& \text { cuando } r \neq 0 \text { y }_{n} a_{x}=n / 2 \text { tenemos: } \\
& l(x+n)=\frac{2{ }_{n} L_{x} e^{-r n / 2}-n l(x)}{n e^{-r n}}
\end{aligned}
$$

Puede observarse que mientras que la fórmula IV.8 no es una solución aproximada sino la exacta, la última (IV.9) constituye una aproximación del mismo tipo que la IV.6.

\section{UN RESUMEN GRÁFICO DE LOS RESUltadoS}

Una manera fácil de resumir los resultados anteriores en tal forma que las interrelaciones entre ellos fueran inmediatamente aparentes es a través de un diagrama de Venn en donde $(A),(B)$, etc., representan los correspondientes conjuntos de fórmulas desarrolladas en la sección IV (véase la gráfica 1). Las soluciones analíticas completas de la transformación de ${ }_{n} L_{x}$ en $l(x)$ se presentan en un apéndice.

\section{GRÁFICA 1}

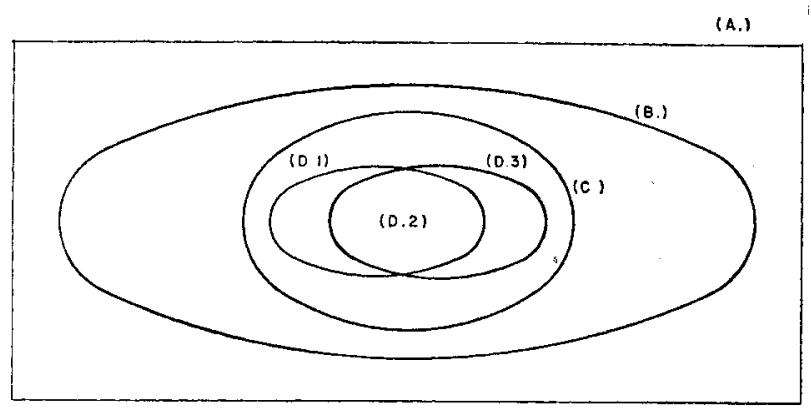




\section{RESUMEN Y CONCLUSIONES}

1. Cada vez que se realiza una interpolación con ${ }_{n} L_{x}$ quedan determinadas por lo menos un par de variables dependientes conjuntas $[l(x)$ y $\left.{ }_{n} a_{x}\right]$. No obstante el hecho de que el resultado deseado de la interpolación convencional es la función $l(x)$, la distribución implícita ${ }_{n} a_{x}$ generada por alguno de tales procedimientos no se ajusta a los "valores esperados" sugeridos por la evidencia empírica. Dado que suponemos que los demógrafos tienen un cierto conocimiento "anterior" acerca del comportamiento del vector ${ }_{n} a_{x}$ en relación con el nivel de la esperanza de vida al nacer, parece importante encontrar una solución al problema de cómo pasar de ${ }_{n} L_{x}$ a $l(x)$ en la cual los valores ${ }_{n} a_{x}$ entren como un insumo explícito. Este ha sido el propósito central de este trabajo.

2. A partir de la definición general del concepto de ${ }_{n} a_{x}$ se han deducido lógicamente una gran variedad de fórmulas. De la solución más general presentada, que cubre el universo completo de transformaciones posibles, (A) hemos llegado al caso de una población con crecimiento cero y distribución lineal de muertes dentro de intervalos de edades, (D.2). No es tarea fácil recomendar la "mejor" fórmula. Su selección deberá ser hecha por cada demógrafo en relación a su conocimiento "previo" de la realidad demográfica que tenga en estudio. Sin embargo, encontramos que una solución intermedia está dada por D.1.

3. Es obvio que la totalidad de este trabajo se basa sobre la exactitud del conocimiento "previo" de los demógrafos acerca de la distribución ${ }_{n} a_{x}$ asociada con la esperanza de vida al nacer, e implícita en las tasas de sobrevivencia ajustadas derivadas de los dos censos. Por otra parte, el conocimiento anotado depende de la relativa invarianza de la distribución de ${ }_{n} a_{x}$. Por fortuna éste parece ser el caso (excepto para $a_{o}$ ) independientemente del nivel de la esperanza de vida al nacer. Sin embargo, sobre este tema clave se requiere investigación adicional.

\section{APENDICE}

RESUMEN DE SOLUCIONES

A. Supóngase que $F(t)$ tiene una primera derivada en $x, x+n$ :

$l(x+n)=\frac{{ }_{n} L_{x} \cdot F(\theta)-{ }_{n} a_{x} \cdot F(o) \cdot l(x)+{ }_{n} L_{x}\left(F^{\prime}(z) \cdot{ }_{n} A_{x}-F^{\prime}(h) \cdot{ }_{n} a_{x}\right)}{F(n) \cdot\left(n-{ }_{n} a_{x}\right)}$

B. Supóngase la condición adicional $F^{\prime}(t)=c \cdot F(t)$, entonces:

$$
l(x+n)=\frac{{ }_{n} L_{x} \cdot F(\theta)-{ }_{n} a_{x} \cdot F(o) \cdot l(x)+c F(\theta) \cdot{ }_{n} L_{x} \dot{\left({ }_{n} A_{x}-{ }_{n} a_{x}\right)}}{F(n)\left(n-{ }_{n} a_{x}\right)}
$$


C. Suponga el caso especial de población estable: $F(t)=K e^{-r t}$,

$l(x+n)=\frac{{ }_{n} L_{x} \cdot e^{-r n / 2}-{ }_{n} a_{x} l(x)+r e^{-r n / 2} \cdot{ }_{n} L_{x} \quad\left({ }_{n} a_{x}-{ }_{n} A_{x}\right)}{e^{-r n / 2}\left(n-{ }_{n} a_{x}\right)}$

D. Considere los siguientes tres casos particulares derivados de (C.):

$$
\text { Cuando } r=o \mathrm{y}_{n} a_{x} \neq n / 2 \text {, entonces: }
$$

$$
l(x+n)=\frac{{ }_{n} L_{x}-{ }_{n} a_{x} l(x)}{n-{ }_{n} a_{x}}
$$

$$
\text { Cuando } r=o \mathrm{y}_{n} a_{x}=\mathrm{n} / 2 \text {, entonces: }
$$

$$
l(x+n)=\frac{2{ }_{n} L_{x}-n \cdot l(x)}{n}
$$

$$
\text { Cuando } r \neq 0 \mathrm{y}_{n} a_{x}=\mathrm{n} / 2 \text {, entonces: }
$$

$$
l(x+n)=\frac{2 \cdot{ }_{n} L_{x} \cdot e^{-m / 2}-n \cdot l(x)}{n \cdot e^{-r n}}
$$

\section{REFERENCIAS BIBLIOGRÁFICAS}

1. G. Mortara, "Methods of Using Census Statistics for the Calculation of Life Tables and other Demographic Measures", Naciones Unidas, Population Studies, Núm. 7, Nueva York, 1949.

2. G. Maccio, "La mortalidad en la república de Cuba, 1943-53 y 1961-63", CELADE, Serie C, Núm. 92, Santiago de Chile, 1967.

3. A. Coale y P. Demeny, "Methods of Estimating Basic Demographic Measures from Incomplete Data", Naciones Unidas, Manual IV, Nueva York, 1967.

4. H. Shryock y J. Siegel, "The Methods and Materials of Demography", Vol. 1 y 2, Departamento de Comercio de los Estados Unidos, Oficina de Censos, Washington, D. C., 1971.

5. H. Beers, "Six-Term Formulas for Routine Actuarial Interpolation", Record of the American Institute of Actuaries, Vol. 34, junio de 1945.

6. A. Bocaz, Notas inéditas, CELADE, 1969.

7. E. Arriaga, "New Life Tables for Latin American Populations in the nineteenth and twentieth Centuries", Population Monograph Series, Núm. 3, Berkeley, University of California, 1968.

8. A. Ortega y J. C. Lerda, "Tablas de mortalidad por generaciones: México, 1960”, Demografía y Economía, Vol. VI, Núm. 1, México, El Colegio de México, 1972.

9. C. Chiang, "Constructing Current Life Tables", Journal of America Statistical Asociation, septiembre de 1972.

10. J. C. Elizaga, "Métodos demográficos para el estudio de la mortalidad", CELADE, Serie E. Núm. 4, Santiago de Chile, 1969.

11. A. Coale y P. Demeny, "Regional Model Life Tables and Stable Populations", Princeton, Princeton University Press, 1966. 
12. N. Keyfitz, "Finding Probabilities from Observed Rates or How to Make a Life Table", The American Statistician, ASA, febrero de 1970.

13. S. Preston, N. Keyfitz, y R. Schoen, "Causes of Death, Life Tables for National Populations", Nueva York y Londres, Seminar Press, 1972.

14. N. Keyfitz, "A Life Table that Agrees with the Data: II", Journal of American Statistical Association, diciembre de 1968.

15. A Taylor y W. Mann, Advanced Calculus, 2a. edición, Lexington, Mass., Xerox College Publishing, 1972. 